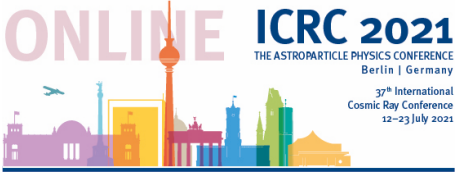

\title{
Bursty betatron acceleration of electrons at nonstationary quasi-perpendicular shocks
}

\author{
Fumiko Otsuka $^{a^{*}}$, Shuichi Matsukiyo ${ }^{a}$, Mitsuo Oka $b$ \\ a Kyushu University, 6-1 Kasuga-Koen, Kasuga, Fukuoka, Japan

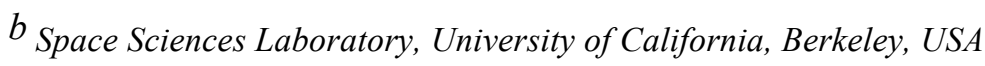 \\ E-mail: otsuka@esst.kyushu-u.ac.jp
}

Energetic, non-thermal electrons with power-law energy spectra are directly observed in the vicinity of the Earth's quasi-perpendicular bow shock. However, the acceleration mechanism of these electrons remains unclear. Here we show, using 1D PIC simulations of a quasi-perpendicular collisionless shock $\left(\mathrm{M}_{\mathrm{A}} \sim 7.1\right.$, shock angle $70^{\circ}$, beta $\left.\sim 0.3\right)$, that bursts of energetic electrons are produced at a reforming magnetic overshoot by betatron acceleration in addition to the shock drift acceleration. The betatron acceleration occurs through efficient trapping in a thin magnetic trough formed by a phase-standing whistler wave, and achieves an energy gain larger than that obtained by the shock drift acceleration only. We also found that the shock potential works against the acceleration, but the accelerations are more efficient and leads to the net energy gain. We envision that this betatron acceleration process is an important component of the entire process of electron acceleration to non-thermal energies at a non-stationary, quasiperpendicular shock.

$37^{\text {th }}$ International Cosmic Ray Conference

12-23 July, 2021

Berlin, Germany

*Speaker

(C) Copyright owned by the author(s) under the terms of the Creative Commons

Attribution-NonCommercial-NoDerivatives 4.0 International License (CC BY-NC-ND 4.0). 


\section{Introduction}

Energetic, non-thermal electrons are directly observed in the vicinity of Earth's quasiperpendicular bow shock [1-3]. The observed electron energy spectra generally show power-law shapes at the shock transition region. In addition, Gosling et al. (1989) [1] showed that the energetic electrons in the upstream region are generally field-aligned beams, whereas they show perpendicular anisotropy in the downstream region. PIC simulations of quasi-perpendicular shocks have reproduced the energetic electrons escaping upstream as field-aligned beams $[4,5]$. Their simulations revealed that these electrons are not reflected uniformly in time. Instead, they are reflected in a bursty manner in association with the nonstationarity of the shock front, i.e., self-reformation. Recent Magnetospheric Multiscale (MMS) observation showed a signature of energetic electron burst at the transition region of the Earth's quasi-perpendicular bow shock [3].

Shock drift acceleration (SDA) is one of the most plausible models to produce non-thermal, field-aligned electron beams [6]. The process is also known as the fast Fermi acceleration [7]. In the SDA, electrons gradient-B drifting along the shock gain energy from the motional electric field. Then, some of the energized electrons are reflected by the converging magnetic field, leading to non-thermal electron beams. If the mirror reflection occurs adiabatically in a stationary shock, the energy is conserved in the so-called de Hoffmann-Teller frame (dHTF), in which the flow of the upstream plasma is parallel to the magnetic field and the motional electric field vanishes. Conversely, the reflected electrons gain energy along the ambient magnetic field in the normal incidence frame (NIF), in which the shock is at rest and the upstream plasma flow is normal to the shock surface. However, it is unclear how much the SDA contributes to the electron energy gain at nonstationary Earth's quasi-perpendicular bow shock. At a self-reforming quasiperpendicular shock where an overshoot of magnetic field magnitude is enhanced periodically, betatron acceleration [8] could also be a candidate for explaining perpendicular energization. Whistler waves excited at the shock transition region also play important roles in electron scattering and confinement near the shock. PIC simulations [5] showed that phase standing, oblique whistler waves are emitted locally in the foot of the quasi-perpendicular shock, and these waves influence the distribution function of the reflected electrons. A cross-shock potential may also influence the energy change of the shock-reflected electrons in a nonstationary shock.

In this paper, we discuss acceleration and reflection processes of electrons at a quasi-perpendicular collisionless shock by performing a one-dimensional particle-in-cell (PIC) simulation. We show an efficient acceleration process of electrons during shock reformation. Some electrons gain energy larger than what is expected from the adiabatic SDA model alone. We found that a bursty betatron acceleration occurs as an additional energy gain process and discuss the effects of a crossshock potential and phase standing whistler waves in the shock transition region. The new acceleration process has an important implication for our understanding of the electron power-law energy spectra frequently observed at Earth's quasi-perpendicular bow shock.

\section{Method and Results}

We perform a one-dimensional particle-in-cell (PIC) simulation in which the plasma and electromagnetic field evolve self-consistently in a space along the $x$-axis, i.e., a shock normal direction. The plasma consists of ions and electrons. The so-called injection method is used to 
produce a collisionless shock. Upstream magnetic field lies in the $x-z$ plane at the shock angle of $70^{\circ}$ from the positive $x$-direction. The other plasma parameters are as follows. The ion-to-electron mass ratio is 625 , the electron plasma frequency is $\omega_{\mathrm{pe}}=10 \Omega_{\mathrm{e}}$, where $\Omega_{\mathrm{e}}$ is electron gyrofrequency. Plasma betas for electrons and ions are 0.15 , respectively. The corresponding thermal velocities are $0.027 c$ and $0.001 c$ for electrons and ions, respectively, where $c$ is the light speed. The time step and spatial grid are $\omega_{\mathrm{pe}} \Delta t=0.025$, and $\Delta x=0.025 \mathrm{c} / \omega_{\mathrm{pe}}$.

A fresh plasma is continuously injected from the left boundary $(x=0)$ with the speed of $u_{\text {in }}=0.02$ $c$. The injected plasma is specularly reflected at the right boundary $\left(x=5000 c / \omega_{\mathrm{pe}}\right)$. A mixture of the injected and reflected plasmas forms a downstream plasma. Hence, the simulation is in the downstream frame of reference. The shock propagates toward the negative $x$-direction with the speed of $-0.0086 c$. The Alfvénic Mach number, $M_{A}$, defined by the upstream bulk flow speed in the shock rest frame, $0.0286 c$, divided by the Alfvén speed of $0.004 c$, is 7.1. In a quasiperpendicular shock, some of the incoming particles are reflected at the shock. The reflected ions form the foot in front of the shock ramp and are convected toward the downstream region, whereas the reflected electrons stream back along the magnetic field due to their gyroradii much smaller than those for ions. The spatial scale of the foot is comparable with the reflected ion gyroradii, $\rho_{\mathrm{in}} / 2$. Here, $\rho_{\mathrm{in}}$ is defined by $\rho_{\mathrm{in}}=\mathrm{u}_{\mathrm{in} /} \Omega_{\mathrm{i} 1}\left(=125 \mathrm{c} / \omega_{\mathrm{pe}}\right)$ and $\Omega_{\mathrm{i} 1}$ is ion gyrofrequency far upstream. It is known that a fast shock becomes nonstationary at a higher $\mathrm{M}_{\mathrm{A}}$, and that the magnetic overshoot repeats growth and collapse. This process is known as shock reformation [e.g., 9].

Fig.1(a) shows the evolution of magnetic field magnitude as a function of space, $x \omega_{\mathrm{pe}} / c$, and time, $\omega_{\text {pe }} t$. Periodic behaviors of the magnetic foot and overshoot structures were evident, indicating the presence of the shock reformation process. In our simulation, the reformation time scale was of the order of $\pi / 2 \Omega_{\mathrm{i} 1}$, which is consistent with previous PIC simulations [4,5].
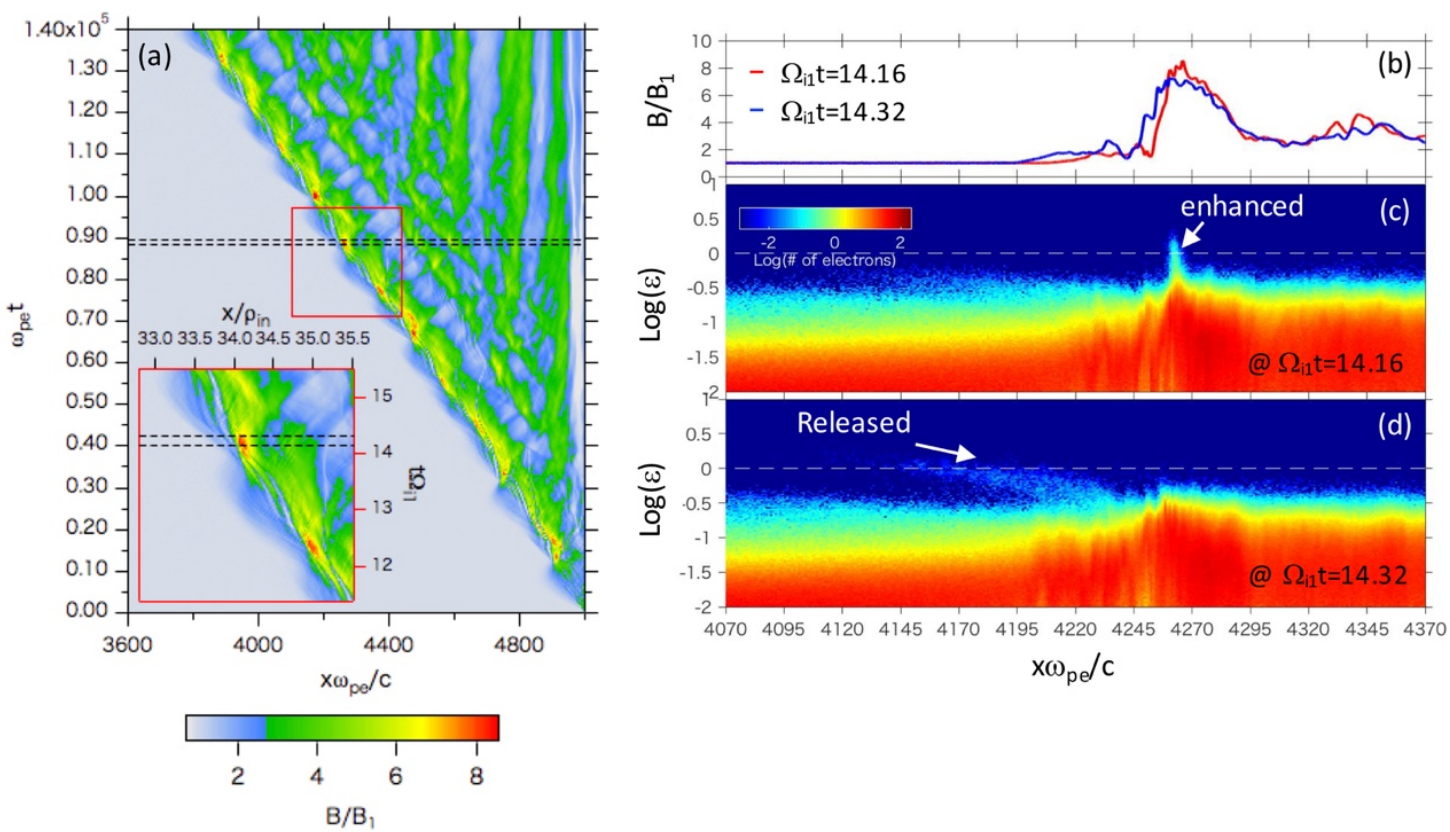

Figure 1. (a) Magnetic field intensity in space and time, (b) Magnetic field intensities at $\omega_{\text {pe }} t=88500$ and $89500\left(\Omega_{\mathrm{i}} \mathrm{t}=14.16\right.$ and 14.32$)$, shown by red and blue lines, respectively. (c, d) Electron energy distribution as a function of space at $\omega_{\text {pe }} \mathrm{t}=88500$ and 89500 , respectively. 
In the inset bounded by the red square, thin magnetic troughs were identified in the foot, which we interpret as a phase standing whistler wave as reported by Matsukiyo and Scholer (2012) [5].

Figure 1(c, d) show the electron energy distributions at $t \omega_{\mathrm{pe}}=88500$ and 89500 , respectively, corresponding to $\Omega_{\mathrm{i} 1} \mathrm{t}=14.16$ and 14.32. Here, the kinetic energy, $\varepsilon$, is defined in the shock rest frame, and normalized to the upstream ion bulk flow energy. When the magnetic overshoot reached its maximum $B=8 B_{1}$ at $\mathrm{t} \omega_{\mathrm{pe}}=88500$, some electrons showed spiky peak in the phase space, well beyond the ion bulk energy of $\varepsilon=1$, as indicated by the white arrow in Fig.1(c). Shortly after that, the magnetic overshoot started to collapse, and these energized electrons were released toward upstream, as indicated by the white arrow in Fig.1(d). These features are consistent with previous simulations $[4,5]$.

The electron energization and release processes occur in a bursty manner. To understand this electron burst process in detail, we analyze a typical trajectory of an accelerated and reflected electron (Fig. 2). It is found from (Fig. 2b, 2c) that the sequence of acceleration and reflection occurs with the approximate time interval of $10000 / \omega_{\text {pe }}$, i.e., $1.6 / \Omega_{\mathrm{i} 1}$, which is equivalent to the shock reformation period. Here, in Panel c, the electron speed is evaluated both in the dHTF and in the NIF.
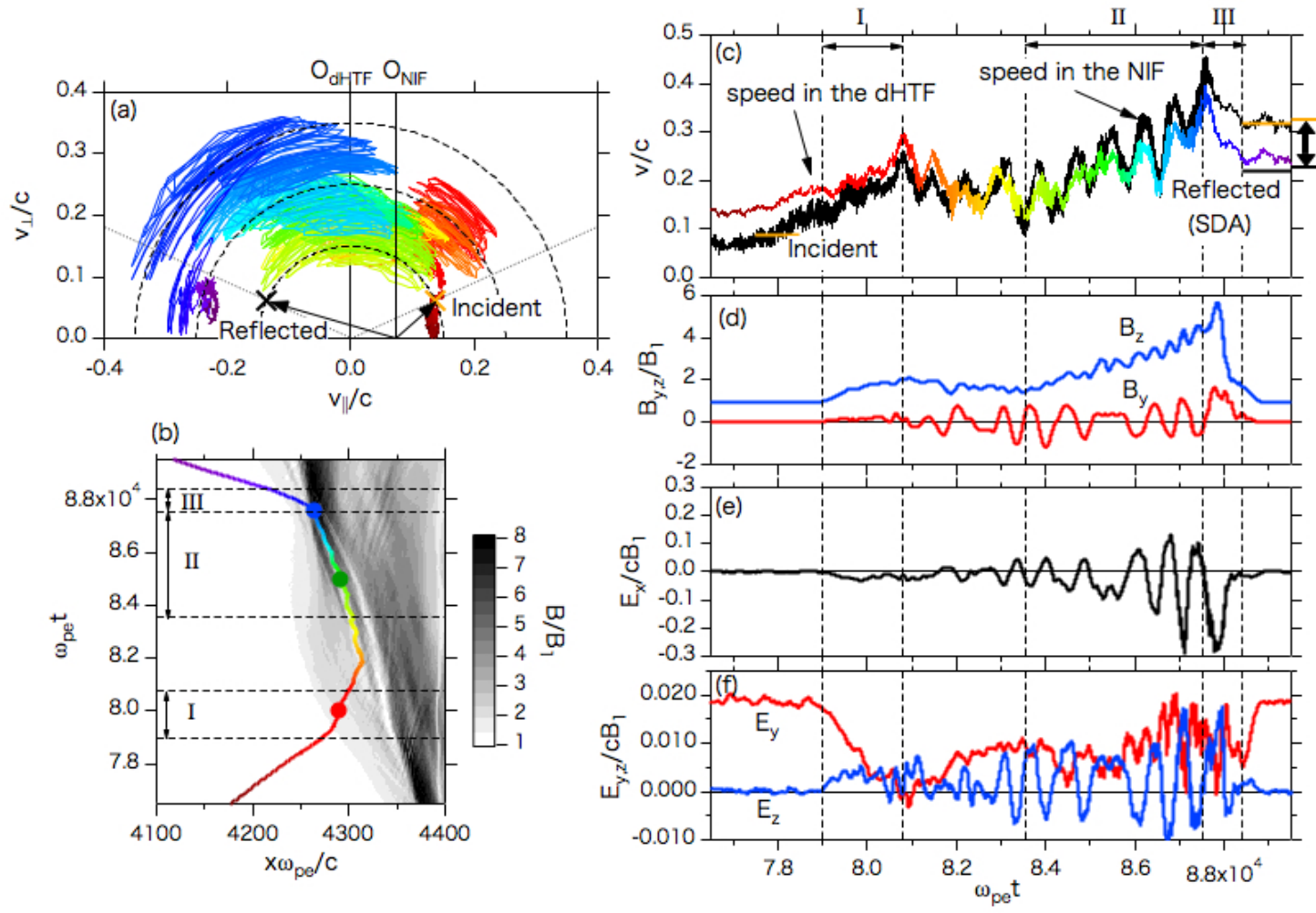

Figure 2. Result for a typical reflected/accelerated electron. (a) Trajectory in the velocities parallel and perpendicular to the upstream magnetic field in the dHTF. (b) Trajectory in space and time. (c) Electron speeds defined in the dHTF and in the NIF showed by the rainbow colored and black curves, respectively. (d-f) Electromagnetic fluctuations experienced by the electron in time. Here, rainbow colors in (a-c) indicate the corresponding time, $t \omega_{\text {pe }}$, shown in (b). In (a), $\mathrm{O}_{\mathrm{dHTF}}$ and $\mathrm{O}_{\mathrm{NIF}}$ represent the origin of the velocity space in the dHTF and NIF, respectively. The dotted lines in (a) represent the critical pitch-angle to reflect in the dHTF. The cross symbols labeled by "Incident" and "Reflected" are for the estimate from the adiabatic SDA. The Roman numerals indicate characteristic three phases (See text in detail). 
After being released from the upstream boundary, the electron was accelerated in the foot region (Fig. 2b) during the time between $t \omega_{\mathrm{pe}}=79000$ and 80800 (hereafter Phase I), as evidenced by the continuous increase of speed (Fig. 2c). Then, the electron was trapped in the thin magnetic trough associated with the standing whistler wave (Fig. 2b) during $t \omega_{\mathrm{pe}}=83550$ and 87500 (hereafter Phase II). During this phase, the electron experienced a substantial increase of the magnetic field, $B_{z}$ (Fig. 2d), and its speed also increased (Fig. 2c). In the velocity space (Fig. 2a), we can see that the particle gained energy in the direction mainly perpendicular to the ambient magnetic field. More specifically, the particle moved roughly along the contour of a constant speed in the velocity space in dHTF, but switched the contour many times as it gained the speed. Finally, the electron slightly lost its energy and was released toward upstream with small pitch-angles when magnetic field rapidly decreased between $t \omega_{\mathrm{pe}}=87500$ and 88400 (hereafter Phase III).

Now we estimate the energy gain by the adiabatic SDA, i.e., an adiabatic mirror reflection in the dHTF. For mirror reflection to occur, the condition $\sin ^{2} \alpha_{1}>B_{1} / B_{2}$ should be satisfied, where $\alpha_{1}$ is a pitch-angle of the upstream electron in the dHTF, $B_{1}$ and $B_{2}$ represent the upstream and downstream magnetic fields, respectively. Here, $B_{2}=5.9 B_{1}$, maximum value experienced by the electron, are used. Then, the critical pitch-angle becomes $\alpha_{1}=24.3^{\circ}$, shown by dotted lines in Fig. 2 (a). The incident velocity shown by the orange cross symbol in (Fig. 2a) is chosen to be satisfied as the pitch-angle equal to $\alpha_{1}$. The speed of the incident velocity is $v=0.088 c$ in the NIF and is shown by the horizontal orange bar around $t \omega_{\mathrm{pe}}=78000 \mathrm{in}$ (Fig. 2c). The velocity after an adiabatic mirror reflection in the dHTF is shown by the black cross mark in (Fig. 2a), and its speed $v=0.22$ $c$ in the NIF is shown by the black horizontal bar around t $\omega_{\mathrm{pe}}=89000$ in (Fig. 2c). In contrast, the numerical data after acceleration shows $v=0.32 c$ in the NIF and it is well above the speed predicted by the adiabatic SDA. Hence, the difference of $v$ shown by the thick black arrows in (Fig. 2c) indicates an additional energy gain achieved by a process other than the SDA. If the process was the adiabatic SDA only, the speed in the dHTF should be constant. Note that the speed $v$ increased when the magnetic field magnitude felt by the particle also increased (Fig. 2d). Thus, we conjecture that another process contributed substantially to the energy gain.

Figure 3 (a) show the electron kinetic energy in the NIF, normalized to the electron rest energy. The energy, averaged over several gyrations, are divided into that of the guiding center motions parallel and perpendicular to the ambient field and that of the rotations around the guiding center, shown by the blue, green, and red curves, respectively. The black curve indicates the sum of these energies. The dotted curve indicates the total energy in the dHTF. The panels (b) and (c) show the magnetic field magnitude felt by the particle and the magnetic moment, $\mu$, respectively. The panel (d) shows the energy increment by the electric field along the $x$ axis, defined as $\Delta \gamma_{\phi}=$ $-\left(e / m_{0 e} c^{2}\right) \int v_{x g} E_{x g} d t$. The value of $\Delta \gamma_{\phi}$ represents the effect of shock potential. Here, the velocity and electric field are averaged over several gyrations. It should be noted that the resolution of the numerical data used here for the time-integration are somewhat low, i.e., $0.2 / \Omega_{\mathrm{e}}$, but sufficiently high to track how the shock potential contributed to the energy gain.

It is found that the net energy gain was $\Delta \mathrm{W}=0.051$ estimated the difference between the initial and the final energies shown by horizontal orange bars (Fig. 3a). Let us look each of the phases 
in detail. In Phase I when the electron was located in the foot region, the energy gain was mainly in the perpendicular gyromotion (red curve) (Fig. 3a) from 0.0067 to 0.025 (3.7 times increase). The particle felt the increasing magnetic field in the foot region (Fig. 3b) with a double increase. Hence, the magnetic moment, $\mu$, was not conserved with 1.9 times increase (Fig. 3c), implying the non-adiabatic betatron acceleration. Also, $\Delta \gamma_{\phi}$ was increased (Fig. 3d). Figure 4(a) and (b), respectively, show the profiles of the magnetic field intensity and electrostatic potential at $\mathrm{t} \omega_{\mathrm{pe}}=80000$ of Phase I. In the foot region, the shock potential has a positive slope, i.e., negative $E_{x}$. Thus, in general, electrons propagating in the positive $x$ direction gains energy.

In Phase II when the electron was located in the overshoot region, the energy gain was mainly in the perpendicular direction (red curve) (Fig. 3a), accompanied by the increased overshoot magnetic field (Fig.3b). Figure 4(c) show the magnetic field intensity profile at $t \omega_{\mathrm{pe}}=85000$ of Phase II. The particle is located at the position shown by the green circle in Fig.2(b) and Fig.4(c). In this phase, the particle was trapped in the thin trough structure embedded in the growing overshoot magnetic field. Figure 4(d) shows the trajectory in the velocity space perpendicular to the upstream magnetic field, $\mathrm{B}_{\mathrm{z}}$, which is the main component of the ambient magnetic field. The rainbow colors in Fig.3(b) and Fig.4(d) indicates the same time in Phase II. The gyroradius in $v_{x^{-}}$ $v_{y}$ plane was gradually increased, accompanied by the increased $B_{z}$ felt by the particle. The perpendicular energy increase was 7.9 times (from 0.0072 to 0.057 ), and the magnetic field increase was 2.8 times. Hence, the magnetic moment was not conserved with 2.9 times increase.

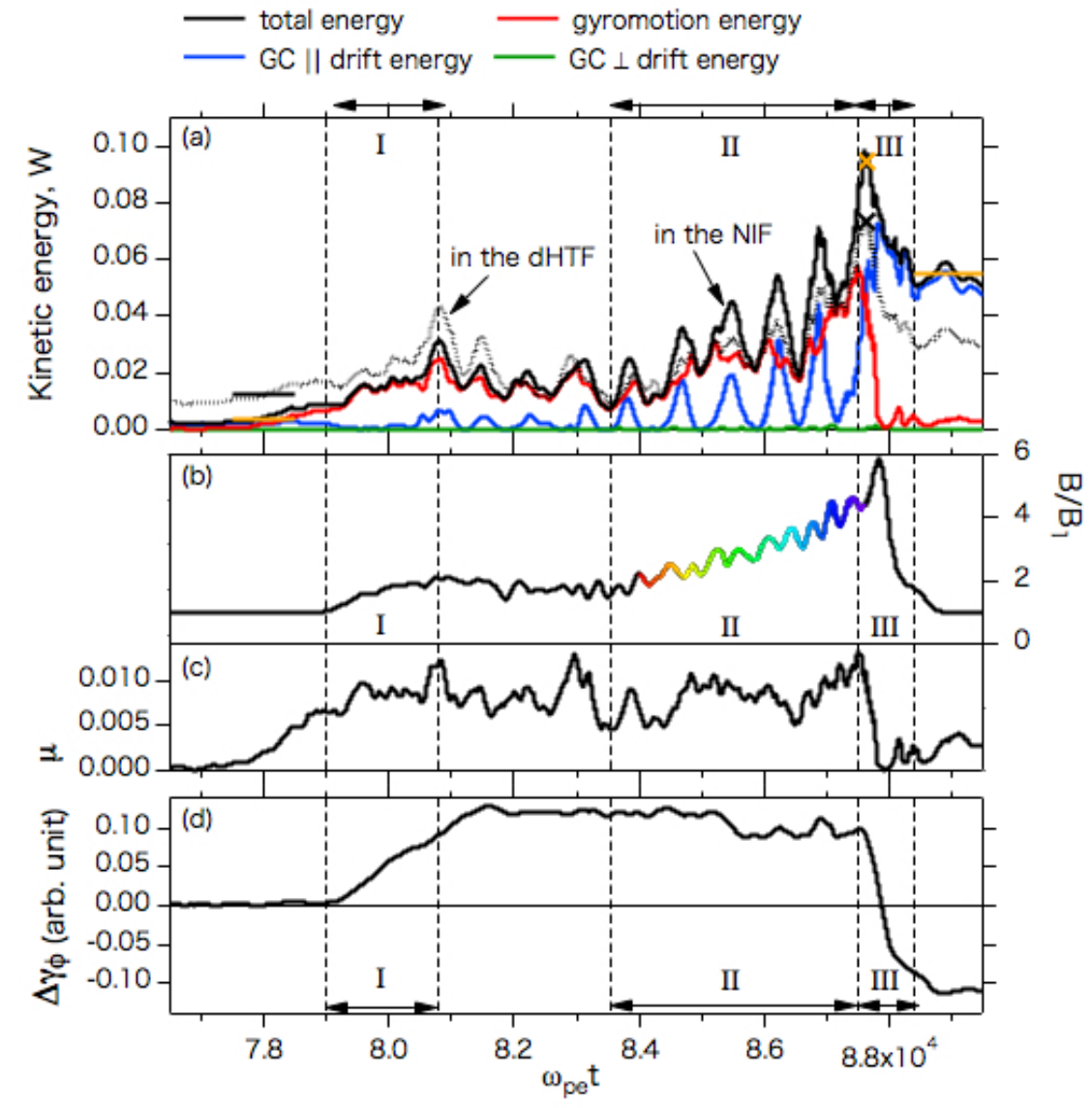

Figure 3. Result of the guiding center (GC) analysis for the electron in Fig.2. (a) Kinetic energies in the NIF (solid curves) for the $\mathrm{GC}$ drift motion and rotations around the GC. The dotted curve indicates the total energy in the dHTF. The energy gain except for the adiabatic SDA is estimated from the difference of the data in the dHTF shown by black horizontal bar and the black cross symbol. (b) Magnetic field intensity felt by the particle. (c) magnetic moment for the particle. (d) energy increment due to the electric field along $x$ axis. The three phases are the same as for Fig.2. 

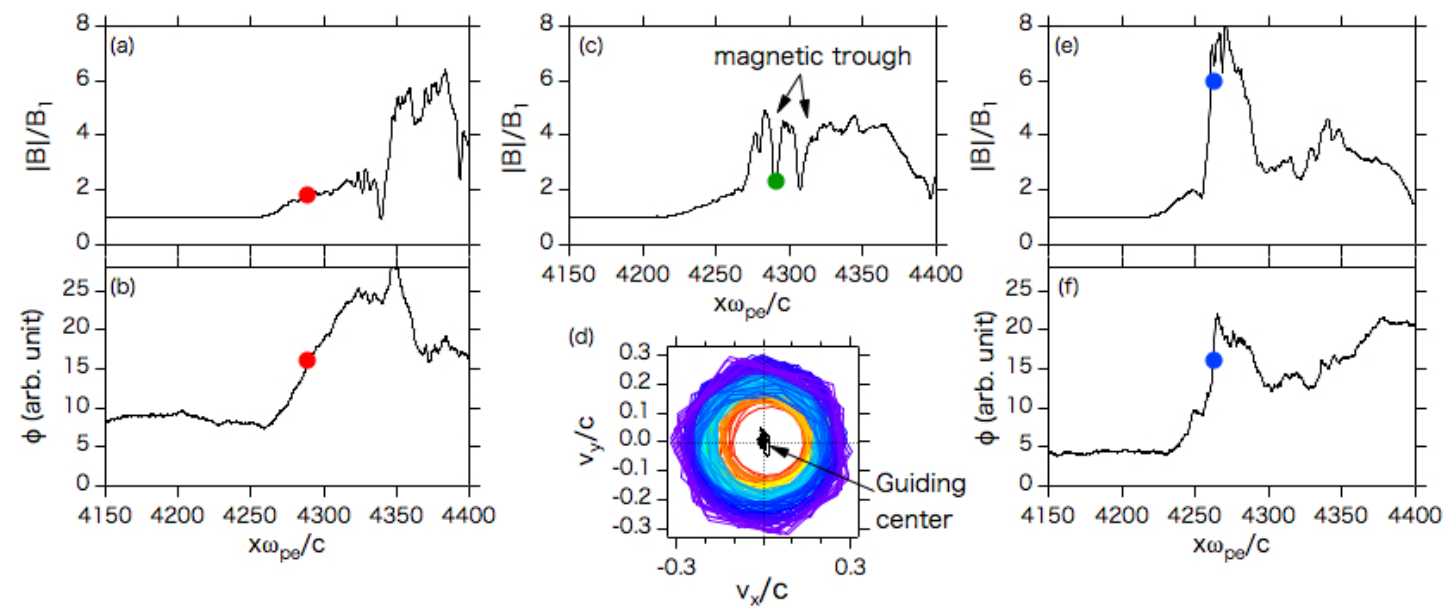

Figure 4. $(a, b)$ Magnetic field intensity and electrostatic potential profile at $t \omega_{\mathrm{pe}}=80000$ of Phase I. (c) Same as the panel (a), but at $\mathrm{t} \omega_{\mathrm{pe}}=85000$ of Phase II. (d) electron gyromotion in velocity space perpendicular to $B_{z}$ component in Phase II. (e,f) Same as the panel $(\mathrm{a}, \mathrm{b})$, but at $\mathrm{t} \omega_{\mathrm{pe}}=87600$ of Phase III. In (a-c, e,f), the colored circles incidate the same particle positions as in Fig.2(b) shown by the same colored circles. In (d), the rainbow colors correspond to the same time as in Fig.3(b).

Thus, we infer that the perpendicular energy gain in Phase II is due to the non-adiabatic betatron acceleration. In addition, the energy of the guiding center motion parallel to the magnetic field (blue curve) let the total energy (black curve) be oscillating. This oscillation reflected the pitchangle scattering in the velocity space (Fig.2a).

In Phase III, the perpendicular gyromotion energy was converted into the parallel drift energy (Fig. 3a) due to the scattering toward the lower pitch-angle (Fig.2a). Simultaneously, the parallel drift energy started to contribute to the increase in the total energy from $t \omega_{\mathrm{pe}}=87155$ (at the end of Phase II) to $t \omega_{\mathrm{pe}}=87860$. This represents the effect of the SDA, because the SDA gives the parallel energy to the shock-reflected electron in the NIF. However, the total energy was decreased from 0.095 to 0.055 which comes predominantly from $\Delta \gamma_{\phi}$ (Fig. 3d). In this phase, the particle was located in the foot region but it has become much narrower (Fig.4f), when compared to Phase I. Hence, the electron moving to the negative $x$-direction quickly lost the energy. As seen from $\Delta \gamma_{\phi}$ (Fig. 3d), the particle lost more energy on its way back to the upstream region (Phase III) than it gained on its way into the shock front (Phase I). Thus, the shock potential act as a decelerator in the whole reflection process. Nevertheless, the amount of energy gain by the betatron acceleration exceeded that of the energy loss from the potential. Therefore, the net energy gain was positive.

Finally, we roughly estimate the energy gain balance from Fig.3(a). The energy gain except for the adiabatic SDA was $\Delta \mathrm{W}_{\text {beta }+ \text { pot }}=0.061$, estimated from the maximum increment of the energy in the dHTF shown by the dotted curve. Then, the energy gain by the adiabatic SDA was $\Delta \mathrm{W}_{\mathrm{SDA}}=0.030$, obtained as the rest of the maximum energy gain, 0.091 , in the NIF (shown by cross orange symbol) minus $\Delta \mathrm{W}_{\text {beta }+ \text { pot }}$. The energy loss by the shock potential was $\Delta \mathrm{W}_{\text {-pot }}=-0.04$, estimated from the decrease of the total energy in Phase III. Hence, the energy gain balance was $\Delta \mathrm{W}=\Delta \mathrm{W}_{\text {beta }+ \text { pot }}+\Delta \mathrm{W}_{\mathrm{SDA}}+\Delta \mathrm{W}_{\text {-pot }}=0.051$. Therefore, the energy gains by the betatron acceleration and the shock potential is found to be twice as large as that estimated from the adiabatic SDA. 


\section{Summary}

We performed the one-dimensional particle-in-cell simulation of quasi-perpendicular collisionless shock to model Earth's bow shock. The shock parameters were Alfvén Mach number of 7.1, shock angle of $70^{\circ}$, plasma beta of 0.3 . Energetic electrons were enhanced at the growing overshoot magnetic field, and they burst out toward the upstream region as the magnetic field decreased. We analyzed the trajectory of an electron reflected during a shock reformation process. The incoming electron was trapped in a thin trough structure embedded in the overshoot magnetic field, related to the standing whistler wave. Simultaneously it gained perpendicular gyration energy via non-adiabatic betatron acceleration while experiencing the growing overshoot magnetic field. The betatron acceleration achieved an energy gain larger than that from the shock drift acceleration(SDA) only. The shock potential led to an energy loss after reflection because of a time variation of the potential structure. However, the sum of energy gains by the betatron acceleration and the SDA exceeded the energy loss by the shock potential, resulting in the net energy gain for the reflected electron. The acceleration time was comparable to the shock reformation period. More quantitative analysis of the electron energization process will be reported soon elsewhere.

\section{Acknowledgements}

This research was partially supported by "Collaborative Research Project on Computer Science with High-Performance Computing in Nagoya University” (F.O.), NASA grants 80NSSC18K1002 and 80NSSC18K1373 at UC Berkeley (M.O.), and Grant-in-Aid for Scientific Research (B) No.17H02966 and (C) No.19K03953 from JSPS (S.M.).

\section{References}

[1] J.T. Gosling, M.F. Thomsen, and S.J. Bame, Suprathermal Electrons at Earth's Bow Shock, J. Geophys. Res., 94, A08, 10,011-10,025 (1989)

[2] M. Oka, T. Terasawa, Y. Seki, M. Fujimoto, Y. Kasaba, H. Kojima, I. Shinohara, H. Matsui, H. Matsumoto, Y. Saito, and T. Mukai, Whistler critical Mach number and electron acceleration at the bow shock: Geotail observation, Geophys. Res. Lett., 33, L24104 (2006), doi:10.1029/ 2006GL028156.

[3] M. Oka, L. B. Wilson III, T. D. Phan, A. J. Hull, T. Amano, M. Hoshino, M. R. Argall, O. Le Contel, O. Agapitov, D. J. Gershman, Y. V. Khotyaintsev, J. L. Burch, R. B. Torbert, C. Pollock, J. C. Dorelli, B. L. Giles, T. E. Moore, Y. Saito, L. A. Avanov, W. Paterson, R. E. Ergun, R. J. Strangeway, C. T. Russell, and P. A. Lindqvist, Electron Scattering by High-frequency Whistler Waves at Earth's Bow Shock, The Astrophys J. Lett., 842:L11 (2017), https://doi.org/10.3847/2041$8213 /$ aa7759

[4] B. Lembege and P. Sovoini, Formation of reflected electron bursts by the nonstationarity and nonuniformity of a collisionless shock front, J. Geophys. Res., 107, ßA3, 1037 (2002)

[5] S. Matsukiyo and M. Scholer, Dynamics of energetic electrons in nonstationary quasiperpendicular shocks, J. Geophys. Res., 117, A11105 (2012), doi:10.1029/2012JA017986

[6] M.M. Leroy and A. Mangeney, A theory of energization of solar wind electrons by the Earth's bow shock, Ann.Geophys., 2, 4, 449-456 (1984)

[7] Wu, C. S., A fast Fermi process: Energetic electrons accelerated by a nearly perpendicular bow shock, J. Geophys. Res., 89, 8857-8862 (1984)

[8] T.G. Northrop, Adiabatic Charged-Particle Motion, Reviews of Geophys., 1, 283 (1963)

[9] A. Balogh, R.A. Treumann, Physics of Collisionless Shocks, ISSI Scientific Report Series 12, Chapter 6, Springer New York Heidelberg Dordrecht London (2013). 